\title{
Interfacial conditions between a pure fluid and a porous medium: implications for binary alloy solidification.
}

\author{
By M. LE BARS AND M. GRAE WORSTER \\ Department of Applied Mathematics and Theoretical Physics, Wilberforce Road, Cambridge, \\ CB3 0WA, United Kingdom
}

(Received 5 July 2006)

The single-domain, Darcy-Brinkman model is applied to some analytically tractable flows through adjacent porous and pure-fluid domains and is compared systematically with the multiple-domain, Stokes-Darcy model. In particular, we focus on the interaction between flow and solidification within the mushy layer during binary alloy solidification in a corner flow and on the effects of the chosen mathematical description on the resulting macrosegregation patterns. Large-scale results provided by the multiple-domain formulation depend strongly on the microscopic interfacial conditions. No satisfactory agreement between the single- and multiple-domain approaches is obtained when using previously suggested conditions written directly at the interface between the liquid and the porous medium. Rather, we define a viscous transition zone inside the porous domain, where Stokes equation still applies, and we impose continuity of pressure and velocities across it. This new condition provides good agreement between the two formulations of solidification problems when there is a continuous variation of porosity across the interface between a partially solidified region (mushy zone) and the melt.

\section{Introduction}

Simultaneous flow through both a pure fluid and a porous medium occurs in a wide range of industrial processes and natural phenomena. It happens for instance during the solidification of multi-component melts, where the solid and the remaining liquid are separated by a layer of mixed phase called a mushy layer that continuously evolves because of internal solidification and local dissolution (see reviews by Worster 1997, 2000, for example).

A classical approach to such systems (either with a reacting or a non-reacting porous matrix) consists of solving the Navier-Stokes equations in the fluid and Darcy's equation in the porous medium (e.g. Levy \& Sanchez-Palencia 1975; Schulze \& Worster 1999). The problem then remains in defining relevant boundary conditions at the interface between the two domains. Continuity of pressure and normal velocity (i.e. mass conservation) are robust and generally accepted boundary conditions. Then, naïve choices regarding the tangential velocity component would be either that it vanishes for low permeability or that it is continuous for large permeability. However, both turn out to be inaccurate, as shown for instance by the experiments of Beavers \& Joseph (1967). Rather, these authors postulated a discontinuity in the interfacial tangential velocity given by

$$
u_{S}-u_{D}=\frac{\sqrt{\Pi}}{\alpha} \frac{\partial u_{S}}{\partial z},
$$

where $z$ is the direction perpendicular to the interface, $u_{S}$ the Stokes velocity calculated 
inside the fluid, $u_{D}$ the Darcy velocity calculated inside the porous matrix and $\Pi$ its permeability. The dimensionless coefficient $\alpha$ characterizes the structure of the permeable material near its interface with the pure liquid, and must therefore be determined for each particular system. This boundary condition was validated experimentally by Beavers \& Joseph (1967) in the case of a Poiseuille flow in a channel formed by an impermeable wall at $z=+h$ and a permeable wall at $z=0$ (cf. figure 1 ). It was also justified analytically by Saffman (1971) using ad hoc representations of forces and energy exchanges at the interface. Since then, more complicated multiple-domain formulations have been suggested, introducing a fluid-fluid viscous correction in the porous matrix (Brinkman 1947). Many types of interfacial conditions have been used, e.g. continuity of the tangential velocity but discontinuity of the tangential shear stress (Ochoa-Tapia \& Whitaker 1995a), or continuity of both the tangential velocity and the tangential shear stress (Neale \& Nader 1974), or discontinuity of both the tangential velocity and the tangential shear stress (Cieszko \& Kubik 1999). Clearly the definition of practical and relevant first-order interfacial conditions between a pure fluid and a porous matrix remains an open question.

To avoid these problems computationally, some models of binary alloy solidification use a single formulation for the solid, the liquid and the mushy layer, which are then resolved on the same mesoscopic scale (see for instance Felicelli et al. 1991; Schneider et al. 1997). This approach eliminates the need for explicit consideration of interfaces. Mesoscopic transport equations are either postulated using mixture theory (Hills et al. 1983) or derived from the volume-averaging of the classical microscopic equations (Beckermann \& Viskanta 1988). Until now, this single-domain method has been mostly utilised to determine the various characteristic fields (temperature, concentration, liquid fraction, velocity) at a mesoscopic scale in industrial settings (e.g. Gu \& Beckermann 1999), whereas the multiple-domain method has been utilised to study the dynamics of interactions between the different regions in idealized theoretical cases (e.g. Chung \& Worster 2002). Results from the two approaches have been compared in the case of the linear stability analysis of double-diffusive convection in superposed fluid and porous layers (Zhao \& Chen 2001).

In the present paper, we apply the single-domain formulation to some analytically tractable cases and systematically compare it with the multiple-domain formulation, using previously suggested interfacial conditions as well as a new set proposed here. Our purposes are to illustrate how large-scale results are influenced by the small-scale structure assumed of the interface and to suggest under which conditions results provided by both approaches agree. Our main interest stems from concerns in modelling interactions between flow and solidification within mushy layers.

In section 2, we derive the single-domain equation of motion (i.e. Darcy-Brinkman) using the volume-averaging method, in a context in which all variables change on scales larger than the averaging length: we highlight the underlying assumptions, define the relevant parameters and variables, and study its limits in the case of either a pure fluid or a small Darcy number, where it respectively gives rise to the Navier-Stokes and Darcy's equations. In section 3, we solve the Darcy-Brinkman equation in the configuration studied by Beavers \& Joseph (1967): a Poiseuille flow in a fluid overlying a porous layer with a constant porosity. This allows us to define a new set of conditions at the mushy layer-liquid interface, which leads to a closer agreement with the experiments of Beavers \& Joseph (1967) compared to the previously proposed interfacial conditions. In section 4 , we similarly study a corner flow in a fluid overlying a porous layer, respectively with a constant porosity and with a linear porosity variation. In addition to the previous case, these self-similar configurations allow us, still with one-dimensional calculations, to take 
into account the effects of flow crossing the interface. Differences between the various formulations are then highlighted. Finally, in section 5, we study the solidification of a binary alloy in a corner flow. The interaction between flow and solidification within the mushy region is precisely examined in this simple geometry, and the importance of the interfacial conditions is highlighted by comparing their effects on the computed macroscopic fields (i.e. temperature, velocity, porosity and, especially, bulk concentration).

\section{Darcy-Brinkman equation and its limits}

The Darcy-Brinkman equation has been widely used to study flows in porous media in various contexts. However, a carreful look at the publications over the past twenty years shows no general agreement regarding its conditions of applicability and the definition of its variables, especially the "effective" viscosity and the relevant pressure. Hence, we present here a concise derivation of the Darcy-Brinkman equation based on the volumeaveraging method, focusing on the underlying assumptions and on its relationships with Navier-Stokes and Darcy's equations. We focus our attention to cases in which all variables are continuous at the mesoscopic scale (i.e. for instance in solidification problems). A complete mathematical description of cases in which the porosity is discontinuous can be found in Ochoa-Tapia \& Whitaker (1995a).

\subsection{A volume-averaged mesoscopic momentum-transport equation}

We consider a domain comprising both a solid and a liquid phase, for instance a porous medium or a solidifying material, and we define a mesoscopic volume $\Delta V$ large enough to smooth the morphological complexities, but small enough to capture the global transport properties (i.e. typically a few pore lengths). We denote by $\eta$ the viscosity of the liquid, $\chi$ the liquid volume fraction (porosity) and $\rho_{k}$ the density of the liquid $(k=l)$ and the solid $(k=s)$ respectively. For simplicity, we suppose that the solid phase is stationary, i.e. $\mathbf{u}_{\mathbf{s}}=\mathbf{0}$, and we neglect variations of material properties inside the control volume $\Delta V$, i.e. $[\eta]^{l}=\eta$ and $\left[\rho_{k}\right]^{k}=\rho_{k}$, where $\left[\rho_{k}\right]^{k}$ stands for the intrinsic volume average in phase $\mathrm{k}$ (see appendix A).

The microscopic momentum equation inside the fluid phase is the Navier-Stokes equation

$$
\frac{\partial}{\partial t} \rho_{l} \mathbf{u}_{\mathbf{l}}+\nabla \cdot\left(\rho_{l} \mathbf{u}_{\mathbf{l}} \mathbf{u}_{\mathbf{l}}\right)=-\nabla P_{l}+\nabla \cdot \mathbf{T}+\rho_{l} \mathbf{g},
$$

where $\mathbf{u}_{\mathbf{l}}$ is the liquid velocity, $P_{l}$ the pressure inside the liquid, $\mathbf{g}$ the acceleration due to gravity, and $\mathbf{T}$ the viscous stress tensor. We consider here a Newtonian fluid, where

$$
\mathbf{T}=\eta\left(\nabla \mathbf{u}_{\mathbf{l}}+\left(\nabla \mathbf{u}_{\mathbf{l}}\right)^{T}\right)
$$

The mesoscopic momentum equation can be obtained by taking the average of (2.1) over $\Delta V$, using concepts and formulas defined in appendix A. Hence,

$$
\frac{\partial}{\partial t}\left[\rho_{l} \mathbf{u}_{\mathbf{l}}\right]+\nabla \cdot\left(\chi\left[\rho_{l} \mathbf{u}_{\mathbf{l}}\right]^{l}\left[\mathbf{u}_{\mathbf{l}}\right]^{l}\right)=-\left[\nabla P_{l}\right]+\nabla \cdot[\mathbf{T}]+\left[\rho_{l} \mathbf{g}\right]+M_{l}+A_{l}+I_{l},
$$

where

$$
\begin{gathered}
M_{l}=-\nabla \cdot\left[\hat{\mathbf{u}}_{\mathbf{l}} \hat{\mathbf{u}}_{\mathbf{l}}\right] \\
A_{l}=\frac{1}{\Delta V} \int_{\Delta A} \mathbf{u}_{\mathbf{l}}\left(\mathbf{w}-\mathbf{u}_{\mathbf{l}}\right) \cdot \mathbf{n}_{\mathbf{l}} d A
\end{gathered}
$$




$$
I_{l}=\frac{1}{\Delta V} \int_{\Delta A} \mathbf{T} \cdot \mathbf{n}_{\mathbf{l}} d A .
$$

Here, $\left[\mathbf{u}_{\mathbf{l}}\right]$ stands for the average value of the liquid velocity over $\Delta V, \hat{\mathbf{u}}_{\mathbf{l}}$ for its fluctuating component, $\mathbf{w}$ for the velocity of the microscopic interface and $\Delta A$ for the interfacial area between the liquid and solid phases in $\Delta V$ (see appendix A).

We can simplify the various terms on the right-hand side as follows. According to formula 3 in appendix A,

$$
\left[\nabla P_{l}\right]=\chi \nabla\left[P_{l}\right]^{l}+\frac{1}{\Delta V} \int_{\Delta A} \hat{P}_{l} \mathbf{n}_{\mathbf{l}} d A
$$

and we neglect the second term, corresponding to pressure fluctuations on the solid-liquid interface. We want to highlight here that $\left[P_{l}\right]^{l}$ (i.e. the average value of the liquid pressure in the liquid only) is the relevant physical parameter: it could for instance be measured experimentally by placing an open pipe filled with pure fluid within the porous structure. Hence, at an interface of a porous matrix, we expect this pressure to be continuous with the pressure in the adjacent pure fluid.

Also from formula 3 of appendix A,

$$
\left[\eta \nabla \mathbf{u}_{\mathbf{l}}\right]=\eta \nabla\left[\mathbf{u}_{\mathbf{l}}\right]+\frac{\eta}{\Delta V} \int_{\Delta A} \mathbf{n}_{\mathbf{l}} \mathbf{u}_{\mathbf{l}} d A
$$

Here, the second term on the right-hand side accounts for the influence of the liquid-solid interface geometry on the large-scale momentum diffusion. When $\mathbf{u}_{\mathbf{l}}$ is uniform, it should be zero, so this term is related to leading order by $\nabla\left[\mathbf{u}_{\mathbf{l}}\right]$. Taking it proportional to $\nabla\left[\mathbf{u}_{\mathbf{l}}\right]$ as a first-order approximation, one can define an effective viscosity $\eta^{*}$ as

$$
\left[\eta \nabla \mathbf{u}_{\mathbf{l}}\right]=\eta^{*} \nabla\left[\mathbf{u}_{\mathbf{l}}\right]
$$

(e.g. Shyy et al. 1997). Hence,

$$
\nabla \cdot[\mathbf{T}]=\nabla \cdot \eta^{*}\left(\nabla\left[\mathbf{u}_{\mathbf{l}}\right]+\left(\nabla\left[\mathbf{u}_{\mathbf{l}}\right]\right)^{\mathbf{T}}\right) .
$$

However, in all the following, we consider either a fixed non-reacting solid matrix or a mushy layer in which $\rho_{l}=\rho_{s}$. Then the liquid velocity at the microscopic liquid-solid interface is zero and (2.6) implies $\eta^{*}=\eta$. In these cases, the effective viscosity should not be taken as an adjustable coefficient, conveniently chosen to match expected results.

The term $M_{l}$ appears because $\mathbf{u}_{\mathbf{l}}$ fluctuates inside the control volume and thus differs from its averaged value. Following Large-Eddy-Simulation techniques in turbulence (e.g. Lesieur 1993; Moin et al. 1991), these fluctuations could be parameterised and related to the mesoscopic gradient of velocity (e.g. Shyy et al. 1997). For simplicity however, we suppose here that the velocity fluctuations remain small and neglect $M_{l}$.

The advective term $A_{l}$ is due to the relative motion of the interface in a reacting porous medium. It appears for instance during solidification, where it is related to the volume change (i.e. to the difference between $\rho_{l}$ and $\rho_{s}$ ), which we neglect in the following.

The interfacial viscous stress exchange $I_{l}$ corresponds to the microscopic momentum exchange of the Newtonian fluid with the solid matrix and must be related to the viscosity of the fluid, to the relative velocity of the solid and the liquid and to the morphology of the porous matrix. As a first approximation, this term can thus be written (Ochoa-Tapia \& Whitaker 1995a)

$$
I_{l}=-\eta\left[\mathbf{u}_{\mathbf{l}}\right] f
$$

where $f$ is some function of the morphology of the porous matrix. Since $I_{l}=0$ in pure fluid,

$$
f \rightarrow 0 \quad \text { when } \quad \chi \rightarrow 1
$$


For comparison with the standard Darcy's law, we will see in section 2.2 that it is convenient to write

$$
I_{l}=-\frac{\eta \chi}{\Pi(\chi)} \mathbf{u}
$$

where the function $\Pi$ introduced here indeed corresponds to the permeability defined in Darcy's law and $\mathbf{u}$ is the Darcy velocity, defined as the volume flow rate per unit cross-sectional area. Hence with our notations,

$$
\mathbf{u}=\left[\mathbf{u}_{\mathbf{l}}\right]=\chi\left[\mathbf{u}_{\mathbf{l}}\right]^{l} .
$$

If we further suppose a mesoscopically incompressible fluid (i.e. $\nabla \cdot \mathbf{u}=0$ ), and if we apply the Boussinesq approximation, the momentum equation finally becomes

$$
\rho_{l} \frac{\partial}{\partial t} \mathbf{u}+\rho_{l} \mathbf{u} \cdot \nabla\left(\frac{\mathbf{u}}{\chi}\right)=-\chi \nabla\left[P_{l}\right]^{l}+\eta \nabla^{\mathbf{2}} \mathbf{u}+\chi \rho_{l} \mathbf{g}-\frac{\eta \chi}{\Pi(\chi)} \mathbf{u},
$$

which is the Darcy-Brinkman equation. Using this equation implies that all fields $\chi,\left[P_{l}\right]^{l}$, $\mathbf{u}$ and $\nabla \mathbf{u}$ are continuous through the whole domain. Although the general form of this equation is well known, variations of it can be found in the literature, especially in the location of the porosity within each term (e.g. Bennon \& Incropera 1987; Shyy et al. 1997; Beckermann et al. 1999; Kaempfer \& Rappaz 2003). Special care should be taken, since having the porosity inside or outside the gradients in 2.13 can lead to large effects when the porosity changes with space, as for instance in a mushy layer.

\subsection{Limits of the Darcy-Brinkman equation}

We can non-dimensionalise (2.13) using a given velocity scale $V$ and a given length scale $L$. If we choose the viscous pressure scale $P=\eta V / L$, then

$$
\operatorname{Re}\left[\frac{\partial}{\partial t} \mathbf{u}+\mathbf{u} \cdot \nabla\left(\frac{\mathbf{u}}{\chi}\right)\right]=-\chi \nabla\left[P_{l}\right]^{l}+\nabla^{2} \mathbf{u}-\chi \frac{\rho_{l} g L^{2}}{\eta V} \mathbf{e}_{\mathbf{z}}-\chi \frac{L^{2}}{\Pi(\chi)} \mathbf{u},
$$

where $\mathbf{e}_{\mathbf{z}}$ is a unit vertical vector and $\operatorname{Re}=\rho_{l} V L / \eta$ the Reynolds number. In the purefluid limit, $\chi=1$ and from (2.10), $\Pi \rightarrow \infty$, so the Darcy-Brinkman equation gives the standard Navier-Stokes equation

$$
\operatorname{Re}\left(\frac{\partial}{\partial t} \mathbf{u}+\mathbf{u} \cdot \nabla \mathbf{u}\right)=-\nabla P+\nabla^{2} \mathbf{u}-\frac{\rho_{l} g L^{2}}{\eta V} \mathbf{e}_{\mathbf{z}},
$$

where $P$ is the liquid pressure.

If we rather choose Darcy's pressure scale $P=\eta L V / \Pi_{0}$ (where $\Pi_{0}$ is a characteristic scale of permability), we obtain

$$
\text { Da } \operatorname{Re}\left[\frac{\partial}{\partial t} \mathbf{u}+\mathbf{u} \cdot \nabla\left(\frac{\mathbf{u}}{\chi}\right)\right]=-\chi\left[\nabla\left[P_{l}\right]^{l}+\frac{\rho_{l} g \Pi_{0}}{\eta V} \mathbf{e}_{\mathbf{z}}+\frac{\Pi_{0}}{\Pi(\chi)} \mathbf{u}\right]+\operatorname{Da}^{\mathbf{2}} \mathbf{u},
$$

where $\mathrm{Da}=\Pi_{0} / L^{2}$ is the Darcy number. In the limit Da $\rightarrow 0$, the Darcy-Brinkman equation gives the standard Darcy's equation

$$
\mathbf{u}=-\frac{\Pi(\chi)}{\Pi_{0}}\left(\nabla\left[P_{l}\right]^{l}+\frac{\rho_{l} g \Pi_{0}}{\eta V} \mathbf{e}_{\mathbf{z}}\right) .
$$

In the following, we study various flows in multiphase domains using both a singledomain approach, where we solve the Darcy-Brinkman equation through the whole domain, and a multiple-domain approach, where we solve Stokes equation in the pure fluid and Darcy's equation in the porous matrix, with relevant interfacial conditions. 


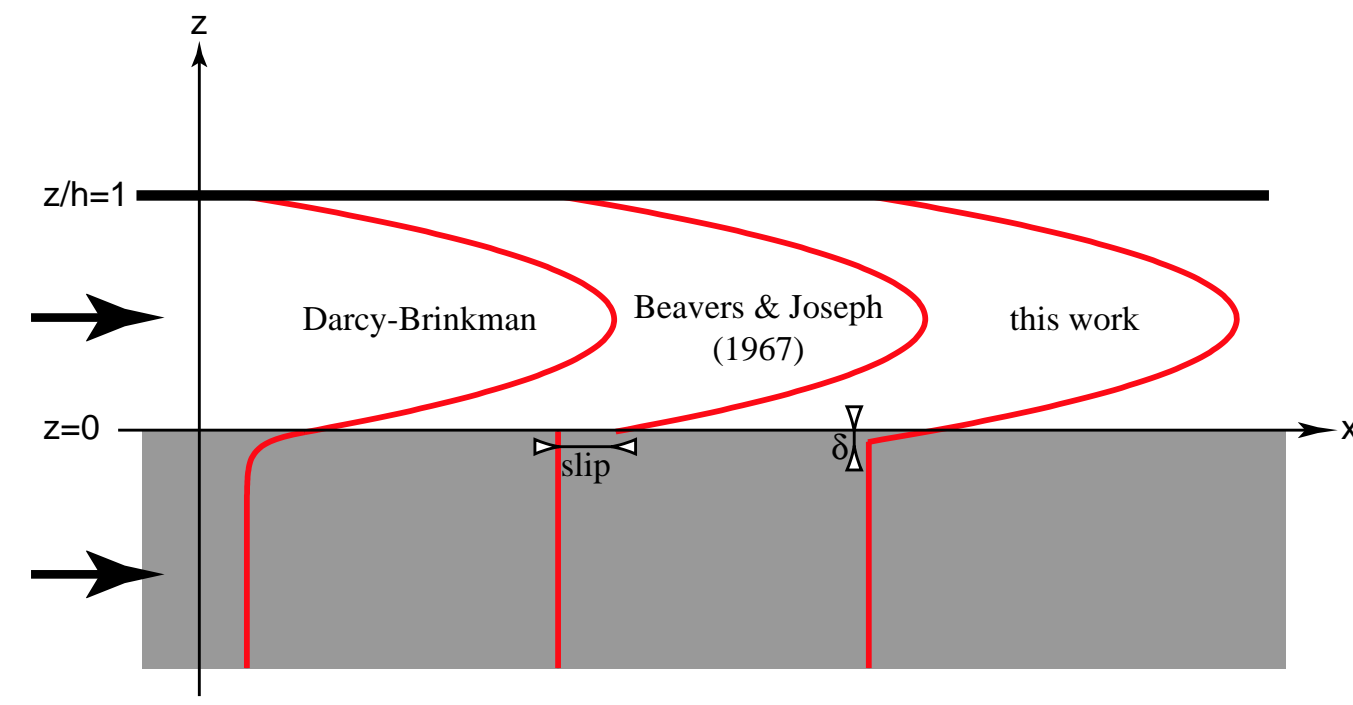

FIGURE 1. Poiseuille flow in a fluid overlying a porous layer: velocity profiles using the limiting Darcy-Brinkman formulation and Stokes-Darcy formulation with either Beavers 83 Joseph (1967) interfacial conditions or the interfacial conditions proposed here. $\chi_{f}=0.5, \Pi\left(\chi_{f}\right) / h^{2}=10^{-3}$ and $\eta^{-1} d p / d x=-1(m . s)^{-1}$.

\section{Poiseuille flow in a fluid overlying a porous layer}

Following Beavers \& Joseph (1967), we consider a two-dimensional Poiseuille flow in a channel formed by an impermeable wall at $z=+h$ and a permeable wall at $z=0$ (cf. figure 1). The boundary at $z=0$ corresponds to the upper surface of a semi-infinite porous medium with a fixed porosity $\chi_{f}$ and permeability $\Pi\left(\chi_{f}\right)$, saturated with the same fluid. A constant horizontal pressure gradient $d p / d x<0$ is imposed through both the liquid and the porous medium, and we look for a velocity in the form $\mathbf{u}=(u(z), 0)$.

\subsection{Darcy-Brinkman formulation}

We first suppose that the fluid in the channel and in the porous medium follows the Darcy-Brinkman equation (2.13). Hence, defining the intrinsic volume average of the dynamic pressure in the liquid $p=\left[P_{l}\right]^{l}-\rho_{l} g z$, we have

$$
0=-\chi \frac{d p}{d x}+\eta \frac{d^{2} u}{d z^{2}}-\frac{\eta \chi}{\Pi(\chi)} u .
$$

In the Darcy-Brinkman formulation, all fields correspond to average values over a mesoscopic volume and are continuous. To better illustrate this point, we suppose the mesoscopic averaging volume to be a cube with side length $\delta_{a}$, typically a few pore lengths. Hence,

$$
\delta_{a}=a \sqrt{\Pi\left(\chi_{f}\right)}
$$

where $a=O(1)$. Then, the liquid fraction is given by $\chi=\chi_{f}$ for $z \leq-\delta_{a}, \chi=1.0$ for $z \geq+\delta_{a}$, and $\chi=\left(1-\chi_{f}\right) z / 2 \delta_{a}+\left(1+\chi_{f}\right) / 2$ for $-\delta_{a} \leq z \leq \delta_{a}$. For a given permeability function following condition (2.10), (3.1) can be solved with the boundary conditions

$$
u(h)=0 \quad \text { and } \quad \frac{d u}{d z}(-\infty)=0 .
$$



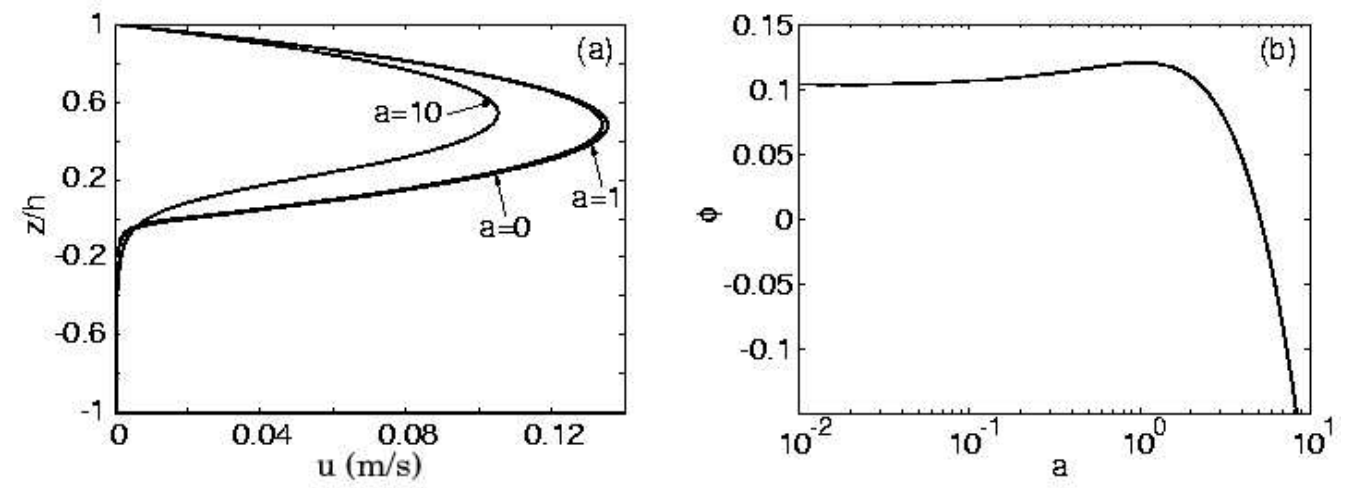

Figure 2. (a): Poiseuille flow profiles computed with Darcy-Brinkman formulation for various values of the averaging size $\delta_{a}=a \sqrt{\Pi\left(\chi_{f}\right)}$. (b): relative flow rate increase depending on the scaling coefficient $a$. In these computations, $\chi_{f}=0.9, \Pi\left(\chi_{f}\right) / h^{2}=10^{-3}$ and $\eta^{-1} d p / d x=-1(m . s)^{-1}$.

For instance, computed profiles are shown in figure $2 a$, using the Carman-Kozeny permeability function (Bear 1972)

$$
\Pi(\chi)=\Pi_{0} \frac{\chi^{3}}{(1-\chi)^{2}},
$$

where $\Pi_{0}$ is a reference permeability.

Computed results depend both on the choice of the permeability function and on the choice of the averaging length $\delta_{a}$. However in all cases, the solution converges towards a single limit when $\delta_{a} \rightarrow 0$, which only depends on the permeability and the porosity of the porous domain. This limit can be determined analytically by solving

$$
\begin{gathered}
0=-\frac{d p}{d x}+\eta \frac{d^{2} u}{d z^{2}} \quad \text { in the liquid } \quad(0<z \leq h), \\
0=-\chi_{f} \frac{d p}{d x}+\eta \frac{d^{2} u}{d z^{2}}-\frac{\eta \chi_{f}}{\Pi\left(\chi_{f}\right)} u \quad \text { in the porous matrix } \quad(-\infty<z<0) .
\end{gathered}
$$

Hence, with $u(h)=0$ and $u$ finite as $z \rightarrow-\infty$,

$$
\begin{gathered}
u(z)=\frac{1}{2 \eta} \frac{d p}{d x}(z-h)(z-A) \quad \text { in the liquid, } \\
u=B e^{z / \delta}-\frac{\chi_{f} \delta^{2}}{\eta} \frac{d p}{d x} \quad \text { in the porous matrix, }
\end{gathered}
$$

where $A$ and $B$ are constants to be determined and

$$
\delta=\sqrt{\frac{\Pi\left(\chi_{f}\right)}{\chi_{f}}} .
$$

Since we use a continuous formulation through both the liquid and the porous matrix, the velocity $u$ as well as its derivative $d u / d z$ must be continuous at $z=0$. This leads to

$$
A=-\frac{h \delta+2 \chi_{f} \delta^{2}}{h+\delta}, \quad B=-\frac{\delta}{2 \eta} \frac{d p}{d x} \frac{h^{2}-2 \chi_{f} \delta^{2}}{h+\delta} .
$$

The lengthscale of the transition zone $\delta$ and the averaging length $\delta_{a}$ are of the same 
order of magnitude. Hence, the limit solution $\delta_{a}=0$ with $\delta \neq 0$ is not mathematically rigorous. Nevertheless, we use it in the following since it is analytically tractable and since it correctly illustrates the behaviour of the full solution (see Ochoa-Tapia \& Whitaker $(1995 b)$ for a complete and mathematically rigorous treatment of this configuration).

\subsection{Comparison with Beavers \& Joseph (1967)}

Following Beavers \& Joseph (1967), we then compute the relative increase in flow rate due to the porous wall

$$
\Phi=\left[-\frac{1}{12 \eta} \frac{d p}{d x} h^{3}\right]^{-1} \int_{0}^{h} u(z) d z-1,
$$

where the denominator corresponds to the flow rate for an impermeable lower wall or for a vanishing tangential velocity at the interface, corresponding to $(3.6 a)$ with $A=0$. In order to compare with Beavers \& Joseph (1967), we define

$$
\alpha=\sqrt{\chi_{f}} \quad \text { and } \quad \sigma=\frac{h}{\sqrt{\Pi\left(\chi_{f}\right)}},
$$

which is the inverse of the square root of the Darcy number. Then, the relative flow rate increase for the limiting case of Darcy-Brinkman is

$$
\Phi=\frac{3(\sigma+2 \alpha)}{\sigma(1+\alpha \sigma)} .
$$

This is exactly the relationship found by Beavers \& Joseph (1967), using Stokes equation in the liquid, Darcy's equation in the porous matrix, and their slip boundary condition at the interface (1.1). Compared to their results, we now have a physical interpretation of the coefficient $\alpha$ for the limiting Darcy-Brinkman case, where $\alpha$ is equal to the square root of the porosity. This agrees with Beavers \& Joseph (1967) conclusion that the coefficient $\alpha$ depends particularly on structural parameters characterizing the nature of the porous surface rather than on the viscosity of the fluid or on the permeability itself.

Taking (3.10), the Darcy-Brinkman velocity and the Beavers \& Joseph (1967) velocity in the pure fluid and deep in the porous matrix are exactly equal. Differences only take place in the viscous transition zone, where the exponential decay in Darcy-Brinkman formulation is replaced by a sharp jump in Beavers \& Joseph (1967).

\subsection{New interfacial conditions between Stokes and Darcy solutions}

In our notation, the Beavers \& Joseph (1967) interface condition (1.1) can be written

$$
u_{D}=u_{S}(0)-\delta \frac{d u_{S}}{d z}=u_{S}(-\delta) .
$$

This can be interpreted as saying that in the mixed Stokes-Darcy approach, the Stokes velocity is extrapolated up to a depth $-\delta$, where it is continuous with the Darcy velocity. Indeed, Darcy's law is a volume-averaged relationship in the porous matrix, and it can not be defined on scales smaller than the averaging length $\delta_{a}$ : in particular, Darcy's law is not valid at a distance closer than $\delta_{a}$ to the interface, and any boundary condition between Stokes and Darcy formulation must be formulated deeper than this.

This conclusion can also be seen in the force balance suggested by the Darcy-Brinkman equation. In the liquid, the pressure gradient is balanced by fluid-fluid interactions (i.e. Stokes equation). Deep in the porous medium, the pressure gradient is balanced by viscous dissipation against the solid matrix (i.e. Darcy's equation). Just below the interface, 
a transition zone exists in which both physical interactions are present. A simple estimation of the forces indicates that Darcy's term becomes larger than the fluid-fluid interaction once

$$
\eta \nabla^{\mathbf{2}} u \sim \eta \frac{u}{z^{2}}<\frac{\eta \chi_{f}}{\Pi\left(\chi_{f}\right)} u
$$

which means

$$
z<-\sqrt{\frac{\Pi\left(\chi_{f}\right)}{\chi_{f}}}=-\delta .
$$

Hence, to leading order, Stokes equation remains valid up to the depth $-\delta$.

In any multiphase-flow study using the mixed Stokes-Darcy formulation, we therefore suggest a generalised interface condition corresponding to continuity of the velocity vector (as well as of the liquid pressure) at the position $z_{i}-\delta$, where $z_{i}$ is the porous-liquid interface position and $\delta$ is the characteristic size of the viscous transition zone (a few pore lengths):

$$
\mathbf{u}_{\mathbf{S}}\left(z_{i}-\delta\right)=\mathbf{u}_{\mathbf{D}}\left(z_{i}-\delta\right) \quad \text { with } \quad \delta=c \sqrt{\frac{\Pi\left(\chi_{f}\right)}{\chi_{f}}}
$$

where $c$ is a constant of order 1 ( $c=1$ for the limiting Darcy-Brinkman case).

This new interfacial conditions is apparently very close to Beavers \& Joseph's (1967) suggestion, and it also necessitates an unknown constant. Nevertheless, it is both physically justified and simpler to implement, especially in numerical simulations. Besides, this new interfacial condition leads to a relative flow rate increase in the Poiseuille geometry of

$$
\Phi=\frac{3(\sigma+2 \alpha+1 / \alpha)}{\sigma(1+\alpha \sigma)},
$$

where $\alpha=\sqrt{\chi_{f}} / c$. As illustrated in figure 3, this equation leads to a slightly better agreement with the experiments by Beavers \& Joseph (1967). We thus expect these interfacial conditions to be more accurate.

We will now test and compare these various formulations in a corner flow, where a vertical flux through the interface also takes place and leads to larger variations.

\section{Corner flow in a fluid overlying a porous layer}

We consider a corner flow in a domain $0 \leq z \leq h, 0 \leq x<\infty$, with pure fluid in the upper half $h / 2<z \leq h$ and a porous matrix of porosity $\chi$ and permeability $\Pi(\chi)$, saturated with the same fluid, in the lower half $0 \leq z<h / 2$ (figure 4). At $z=h$, a purely vertical input velocity $\mathbf{u}=(0,-V)$ is imposed; at the lower wall $z=0$, we suppose no vertical velocity $v=0$ and no horizontal shear $\partial u / \partial z=0$; at the vertical wall $x=0$, we suppose no horizontal velocity $u=0$ and no vertical shear $\partial v / \partial x=0$. We look for the stationary solution and we suppose that the flow is sufficiently slow to neglect inertial effects (i.e. the left-hand side of (2.13) is zero).

Following the well-known results in the case of a pure fluid (e.g. Batchelor 2000), we look for a solution of the form

$$
\mathbf{u}=\left(-x V f^{\prime}(z), V f(z)\right)
$$

which directly satisfies the mass conservation equation $(\nabla \cdot \mathbf{u}=0)$ and the boundary conditions at the vertical wall. We thus look for a function $f$ that satisfies the equations 


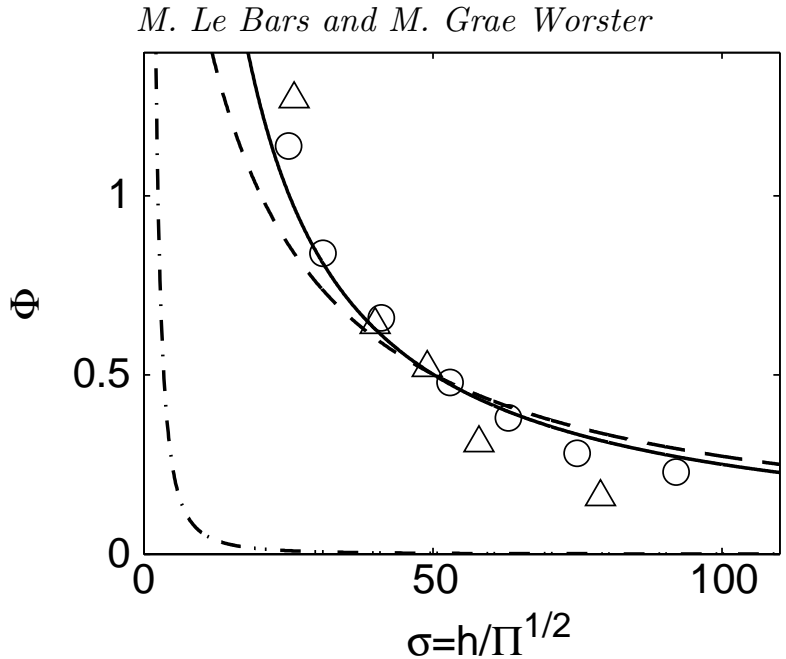

FigURE 3. Relative flow rate increase as a function of the inverse of the square root of the Darcy number. Triangles and circles represent experimental measurements by Beavers \&5 Joseph (1967) using aloxite porous specimens. The continuous line corresponds to the best fit according to (3.16) $(\alpha=0.12)$, using our proposed interfacial conditions (3.15). The dashed line corresponds to the best fit according to (3.11) $(\alpha=0.1)$, using Beavers \&5 Joseph (1967) interfacial conditions (1.1) or the limiting Darcy-Brinkman formulation (see §3.1). The dashed-dotted line corresponds to a continuous velocity at the interface, given by the limit of (3.16) when $\delta \rightarrow 0$, i.e. when $\alpha \rightarrow \infty$. By definition, the condition of zero tangential velocity leads to $\Phi=0$.

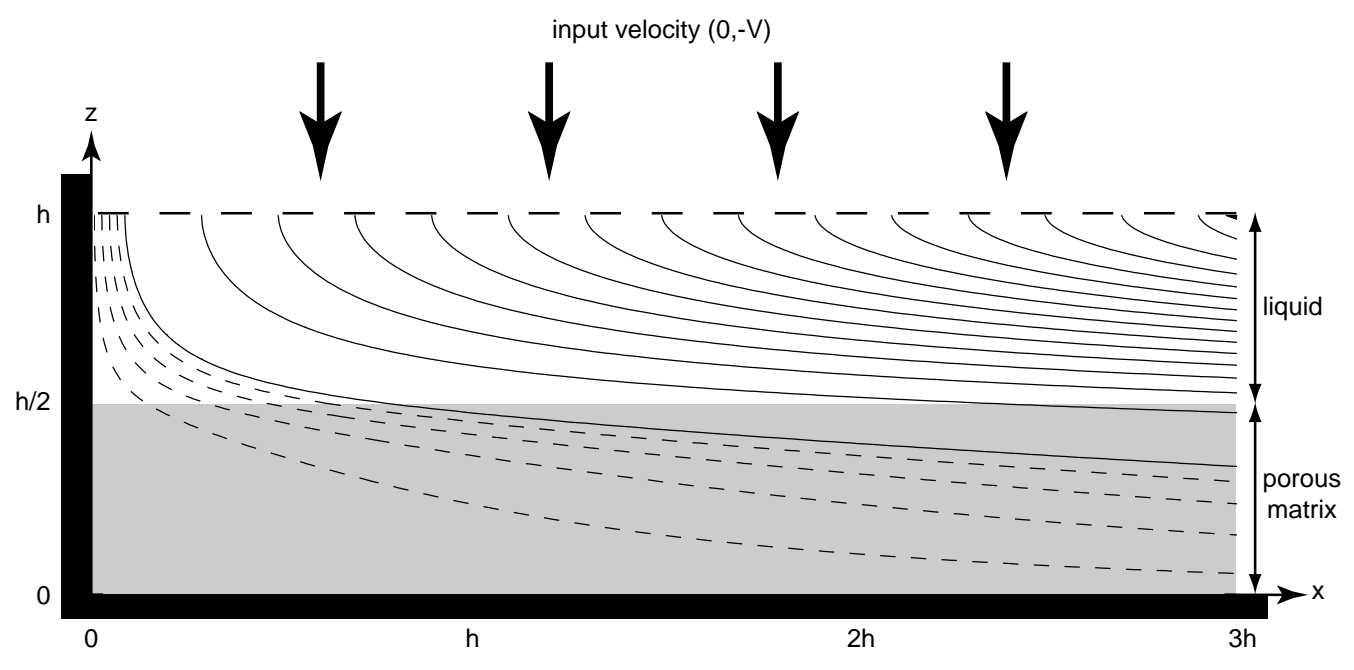

FIGURE 4. Corner flow in a fluid overlying a porous layer with a constant porosity: geometry and streamlines given by the limiting Darcy-Brinkman solution with constant $\chi_{f}=0.2$ and $D a=10^{-3}$. Continuous streamlines are equally spaced from 0.1 to 2.9 with a spacing 0.2 , and dashed streamlines from 0.02 to 0.08 with a spacing 0.02 .

of motion as well as the boundary conditions

$$
f(h)=-1, \quad f^{\prime}(h)=0, \quad f(0)=0 \quad \text { and } \quad f^{\prime \prime}(0)=0 .
$$

In the following, all equations are non-dimensionalised using the input velocity $V$, the depth of the domain $h$ and the viscous pressure scale $\eta V / L$. 


\subsection{Constant porosity}

We first consider that the porous matrix has a constant porosity $\chi_{f}$ and a constant permeability $\Pi\left(\chi_{f}\right)$.

\subsubsection{Darcy-Brinkman formulation}

For $0 \leq z \leq 1$, the fluid follows the Darcy-Brinkman equation

$$
0=-\nabla p+\frac{1}{\chi} \nabla^{\mathbf{2}} \mathbf{u}-\frac{h^{2}}{\Pi(\chi)} \mathbf{u} .
$$

Using (4.1) and eliminating the pressure, we find

$$
0=\frac{d}{d z}\left[\frac{1}{\chi} f^{\prime \prime \prime}-\frac{h^{2}}{\Pi(\chi)} f^{\prime}\right] .
$$

As previously, this equation can be solved for any given averaging size $\delta_{a}$ and permeability function. All solutions converge towards a single limit when $\delta_{a} \rightarrow 0$. Taking into account (4.4) and the fact that $\chi \rightarrow 1$ and $\Pi(\chi) \rightarrow \infty$ in the liquid, $\chi \rightarrow \chi_{f}$ and $\Pi(\chi) \rightarrow \Pi\left(\chi_{f}\right)$ in the porous matrix, the limiting solution can be found analytically by solving

$$
\begin{gathered}
0=f^{\mathrm{iv}} \quad \text { in the liquid } \quad(1 / 2<z \leq 1), \\
0=f^{\mathrm{iv}}-\frac{1}{\delta^{2}} f^{\prime \prime} \quad \text { in the porous matrix } \quad(0 \leq z<1 / 2),
\end{gathered}
$$

where

$$
\delta=\sqrt{\frac{\mathrm{Da}}{\chi_{f}}} \quad \text { and } \quad \mathrm{Da}=\frac{\Pi\left(\chi_{f}\right)}{h^{2}} .
$$

Hence, taking (4.2) into account,

$$
\begin{gathered}
f(z)=-1+A(z-1)^{2}+B(z-1)^{3} \quad \text { in the liquid, } \\
f(z)=C \sinh (z / \delta)+D z \quad \text { in the porous matrix, }
\end{gathered}
$$

where the constants $A-D$ are determined from the interfacial conditions that $f$ and its first two derivatives must be continuous. Additionally, continuity of pressure at the interface requires that

$$
\left.f^{\prime \prime \prime}\right|_{(1 / 2)^{+}}=\frac{1}{\chi_{f}} f^{\prime \prime \prime}-\left.\frac{1}{\mathrm{Da}} f^{\prime}\right|_{(1 / 2)^{-}} .
$$

The solution can be found straightforwardly and is plotted as a dashed curve in figure 5 .

\subsubsection{Mixed Stokes-Darcy formulation}

In the pure liquid domain, the velocity again obeys the Stokes equation $(4.5 a)$, and the function $f_{S}$ is given by $(4.7 a)$. However, in the porous matrix, the velocity follows Darcy's equation

$$
0=-\nabla p-\frac{1}{\mathrm{Da}} \mathbf{u}
$$

which gives

$$
f_{D}^{\prime \prime}=0 \quad \text { with } \quad f_{D}(0)=0 \Rightarrow f_{D}(z)=C z .
$$

The constants $A-C$ are determined by three interfacial conditions between the Stokes and Darcy regions. In all cases, continuity of pressure requires that

$$
-f_{S}^{\prime \prime \prime}=\frac{1}{\mathrm{Da}} f_{D}^{\prime}
$$



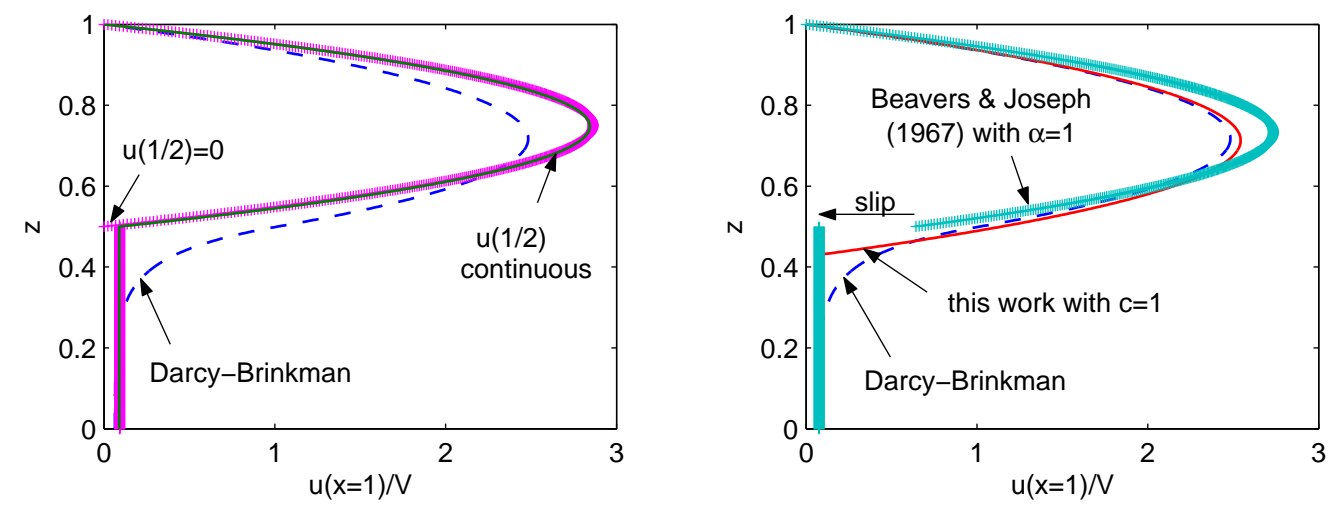

FiguRE 5. Horizontal velocity profiles with $\chi_{f}=0.2, D a=10^{-3}$, for the various proposed interface boundary conditions.

The two addtional constraints depend on the chosen interfacial conditions. For our interfacial conditions (3.15), we introduce the typical size of the viscous transition $\delta$ as

$$
\delta=c \sqrt{\frac{\mathrm{Da}}{\chi_{f}}},
$$

where $c$ is a scaling constant, and write the continuity of the velocity vector at $z_{\delta}=$ $1 / 2-\delta$ :

$$
-1+A\left(z_{\delta}-1\right)^{2}+B\left(z_{\delta}-1\right)^{3}=C z_{\delta} \quad \text { and } \quad 2 A\left(z_{\delta}-1\right)+3 B\left(z_{\delta}-1\right)^{2}=C .
$$

For Beavers \& Joseph (1967) conditions, the continuity in normal velocity and the slip condition (1.1) at $z=1 / 2$ imply that

$$
-1+A / 4-B / 8=C / 2 \text { and } \quad-A+3 B / 4-C=\frac{\sqrt{\mathrm{Da}}}{\alpha}(2 A-3 B),
$$

where $\alpha$ is a constant to be determined.

\subsubsection{Results}

Horizontal velocity profiles for the limiting Darcy-Brinkman formulation as well as for the Stokes-Darcy formulation with various suggested interfacial conditions are presented in figure 5. We also depict in figure 6 the integrated difference between a given formulation and Darcy-Brinkman results, for both the vertical and the horizontal velocities.

Both conditions of continuous horizontal velocity at $z=1 / 2$ and zero horizontal velocity at $z=1 / 2$ are incompatible with Darcy-Brinkman results: they lead to differences of several percent throughout the whole liquid region. Beavers \& Joseph (1967) interfacial conditions as well as our interfacial conditions can be adapted through their respective scaling coefficient, and give a small difference confined to the neighbourhood of the interface only. The best correspondance for both the vertical and the horizontal velocities is obtained with our boundary conditions for $c \sim 1$. The dependence on Darcy number Da is shown in figure 7 for both a continuous tangential velocity at $z=1 / 2$ and our interfacial conditions. In all cases, the difference between Darcy-Brinkman and Stokes-Darcy formulation decreases with the Darcy number, and all formulations are formally consistent in the limit $\mathrm{Da} \rightarrow 0$. Indeed, the flow then focuses into the fluid part of the system only, with a zero velocity in the porous medium. Note however that at $\mathrm{Da}=10^{-6}$, the 

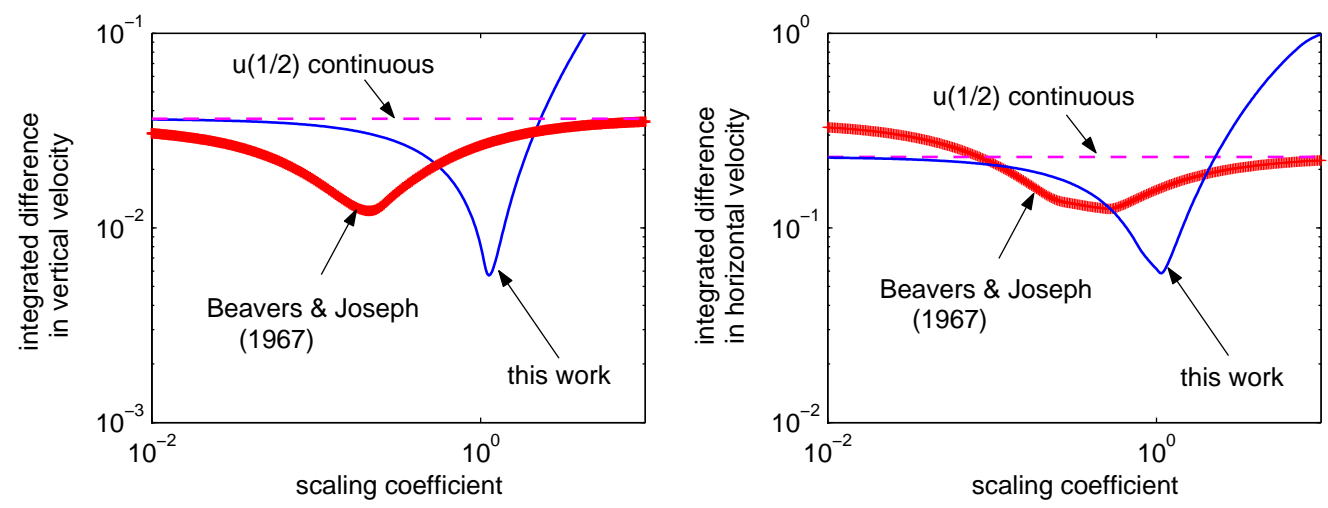

FigURE 6. Integrated difference between mixed Stokes-Darcy and Darcy-Brinkman results as a function of the scaling coefficient (i.e. $\alpha$ for Beavers $\&$ Joseph (1967) and c for our interfacal conditions) for a corner flow in a fluid overlying a porous layer with a constant porosity $\left(D a=10^{-3}\right.$ and $\left.\chi_{f}=0.2\right)$.

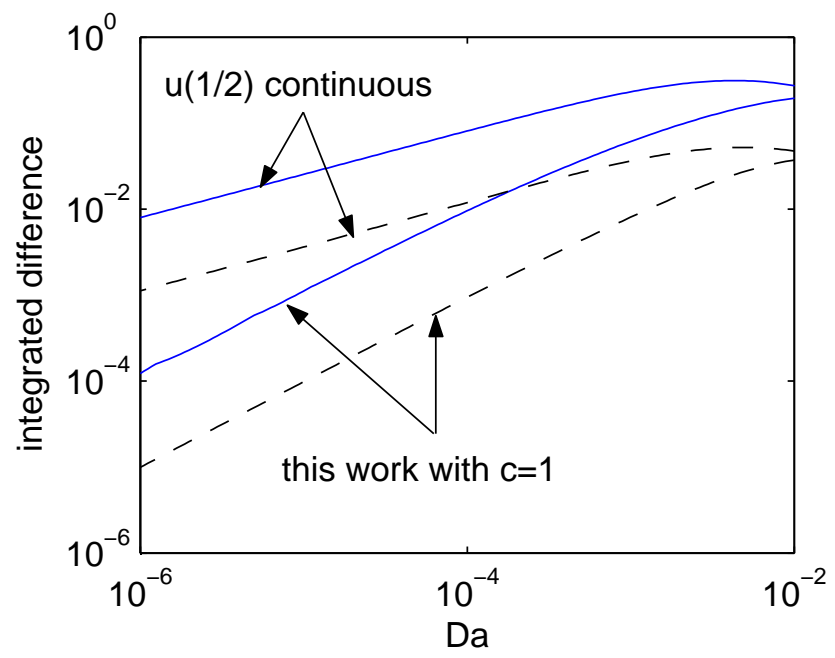

FIGURE 7. Integrated difference in horizontal (continuous line) and vertical (dashed line) velocities between mixed Stokes-Darcy and Darcy-Brinkman results as a function of the Darcy number for $\chi_{f}=0.2$, using our interfacial condition with $c=1$ or a continuous horizontal velocity at $z=1 / 2$.

relative difference is two orders of magnitude smaller when using our interfacial condition compared to continuity of the velocity at $z=1 / 2$.

In figure 8 , we show the relative flow rate increase in the pure fluid, compared to the classical interfacial condition of zero tangential velocity. As opposed to the Poiseuille geometry (see section 3.2), the Darcy-Brinkman method and Beavers \& Joseph (1967) interfacial conditions do not give the same results. Rather, two opposite trends take place: Darcy-Brinkman as well as our interfacial conditions suggest a possible decrease of the flow rate, whereas Beavers \& Joseph (1967) interfacial condition as well as continuity of the velocity always show $\Phi>0$. This suggests an experimental test that could be used to discern between these various formulations. 


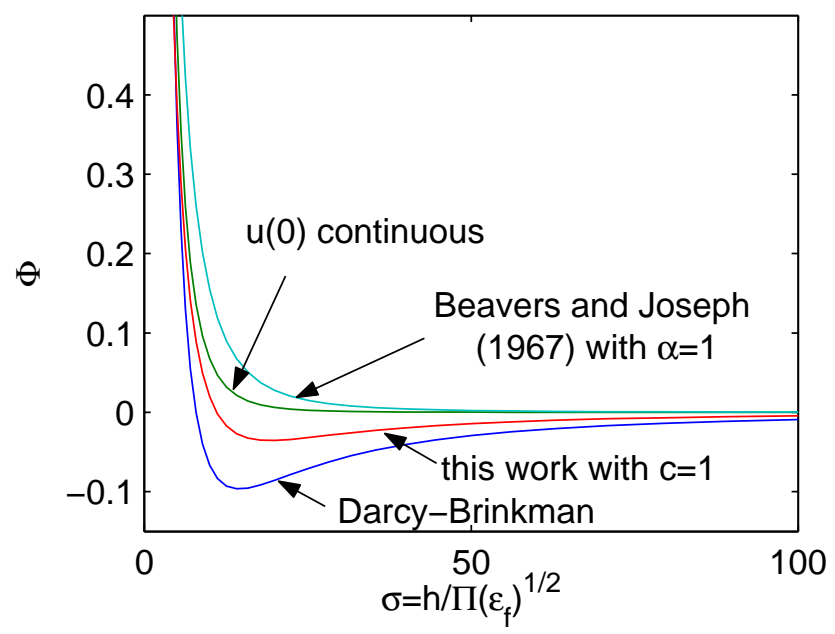

FigURE 8. Relative flow rate increase as a function of the inverse of the square root of the Darcy number with $\chi_{f}=0.2$, for the various proposed interfacial boundary conditions. By definition, the condition of zero tangential velocity leads to $\Phi=0$.

\subsection{Linear porosity}

The previous simple example was solved analytically, so the jump in porosity could be accomodated when solving the Darcy-Brinkman equation. However, the Darcy-Brinkman formulation is most often used in numerical studies of systems in which the porosity exhibits large-scale variations and continuous transition towards the pure fluid (e.g. mushy layers). To investigate the consequences of this, we now consider the same configuration but with a porosity changing linearly from 1 at the interface $z=1 / 2$ to $\chi_{0}$ at $z=0$, i.e.

$$
\chi(z)=\chi_{0}+2\left(1-\chi_{0}\right) z .
$$

\subsubsection{Darcy-Brinkman formulation}

For $0 \leq z \leq 1$, the fluid follows the Darcy-Brinkman equation (4.4), with the porosity given by (4.15) and the Carman-Kozeny relationship (3.4). We solved this ordinary differential equation numerically, subjected to the boundary conditions (4.2). Results are shown in figure 9.

\subsubsection{Mixed Stokes-Darcy formulation}

In the pure liquid domain, the function $f_{S}$ is again given by $(4.7 a)$. In the porous matrix, the velocity follows Darcy's equation. Eliminating the pressure, one finds

$$
\frac{d}{d z}\left[\frac{f_{D}^{\prime}(z)}{\Pi(\chi)}\right]=0
$$

which, using the porosity given by (4.15), the Carman-Kozeny relationship (3.4), and taking into account that $f_{D}(0)=0$, can be solved to give

$$
f_{D}(z)=f_{D}^{\prime}(0) \frac{1-\chi_{0}}{2 \chi_{0}^{3}} G(z)
$$

where

$$
G(z)=\frac{1}{1-\chi_{0}} \frac{2 z}{1-2 z}+3 \ln (1-2 z)+6\left(1-\chi_{0}\right) z+2\left(1-\chi_{0}\right)^{2} z(z-1) .
$$


The three unknown constants $A, B$ and $f^{\prime}(0)$ are determined by the interfacial conditions. When using Carman-Kozeny permeability function, $\Pi \rightarrow \infty$ when $z \rightarrow 1 / 2$. Hence, physically, Darcy's equation is not relevant near the interface $z=1 / 2$. Moreover, mathematically, all interfacial conditions between Stokes and Darcy domains written at $z=1 / 2$ are not usable. We can however use our interfacial conditions, which lead to $f$, $f^{\prime}$ and the pressure being all continuous at a depth $z_{\delta}=1 / 2-\delta$. The latter implies

$$
-f_{S}^{\prime \prime \prime}\left(z_{\delta}\right)=\frac{1}{\mathrm{Da}} \frac{\left(1-\chi_{\delta}\right)^{2}}{\chi_{\delta}^{3}} f_{D}^{\prime}\left(z_{\delta}\right),
$$

where now $\mathrm{Da}=\Pi_{0} / h^{2}$.

The definition of $\delta$ is not as straightforward as in the case of constant porosity, since the porosity and the permeability change with depth. We suggest calculating $\delta$ using (3.15) with the local value of the porosity and the permeability, i.e. non-dimensionally

$$
\delta=c \sqrt{\frac{\Pi\left(z_{i}-\delta\right) / h^{2}}{\chi\left(z_{i}-\delta\right)}},
$$

where $c$ is a scaling constant. With a linear porosity and Carman-Kozeny permeability, this is a polynomial equation in $\delta$. In general, since we expect $\delta$ to be small and $\chi\left(z_{i}-\delta\right)$ to be very close to 1 , a first-order development of (4.20) using (3.4) gives

$$
\delta=\sqrt{\frac{c}{\chi^{\prime}\left(z_{i}\right)}} \mathrm{Da}^{1 / 4} .
$$

\subsubsection{Results}

Typical profiles for Darcy-Brinkman and mixed Stokes-Darcy formulations are presented in figure 9. The integrated difference between the two formulations is shown as a function of the scaling coefficient $c$ in figure 10. In the present case, continuity of velocity at the fluid-porous matrix interface $(c \rightarrow 0)$ gives unrealistic results, where the horizontal velocity is maximum at the interface with a sharp jump between the two domains. This behaviour is mainly due to the divergence of the porosity given by the Carman-Kozeny equation when the liquid fraction goes to 1 . In contrast, the Darcy-Brinkman formulation and our interfacial conditions smooth this discontinuity by introducing a viscous transition layer between the liquid and the porous matrix. Note that $\delta \sim \mathrm{Da}^{1 / 4}$ now rather than $\delta \sim \mathrm{Da}^{1 / 2}$ for constant porosity. Hence, the effects of the viscous layer remain important over a large depth, even for very small Darcy number. The best agreement between the two formulations is obtained for $c \sim 2$.

This simple example illustrates how the choice of the interfacial condition influences the macroscopic computed results. As we will now see, this will be especially important when studying the solidification of a binary alloy, since first-order results regarding macroscopic physical properties (especially the bulk concentration) will depend strongly on the chosen velocity conditions at the interface.

\section{Directional solidification into a corner flow}

Our interest in comparing the single-domain, Darcy-Brinkman model with the multipledomain, Stokes-Darcy model for flows through adjacent porous and pure-fluid domains stems from concerns in modelling interactions between flow and solidification within mushy layers. We therefore conclude by examining a simple problem of alloy solidification, where some of these issues can be confronted. 

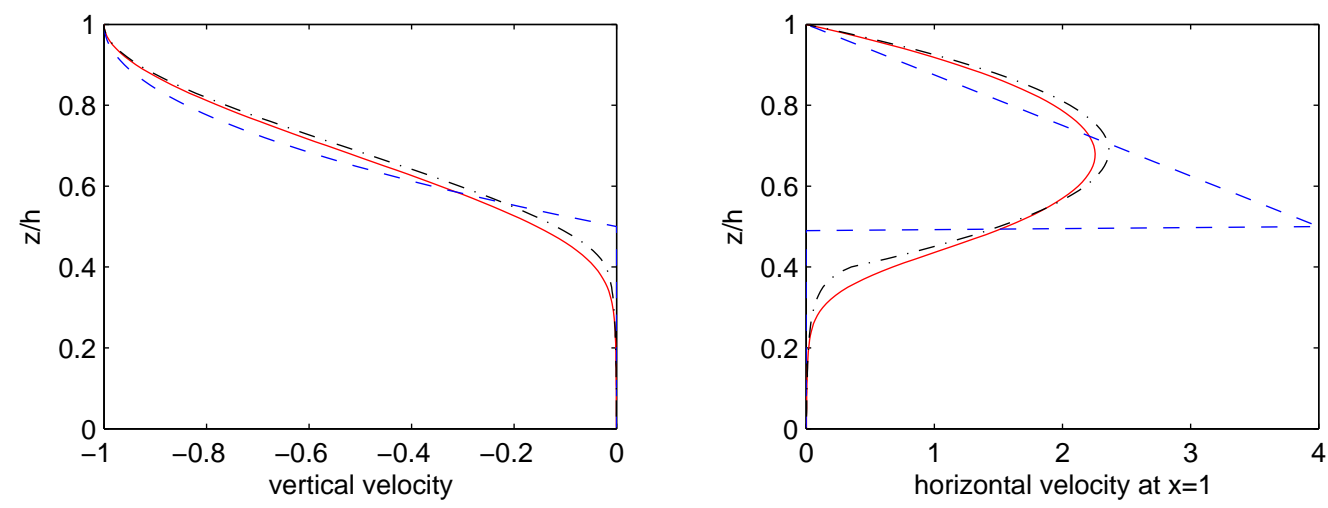

Figure 9. Comparison between mixed Stokes-Darcy and Darcy-Brinkman formulations for a corner flow in a fluid overlying a porous layer with a linear porosity $\left(D a=10^{-4}\right.$ and $\left.\chi_{0}=0.5\right)$. Continuous line: Darcy-Brinkman; dashed line: mixed Stokes-Darcy in the limit $z_{\delta} \rightarrow(1 / 2)^{-}$ (i.e. $c \rightarrow 0)$; dashed-dotted line: mixed Stokes-Darcy with $c=1$.

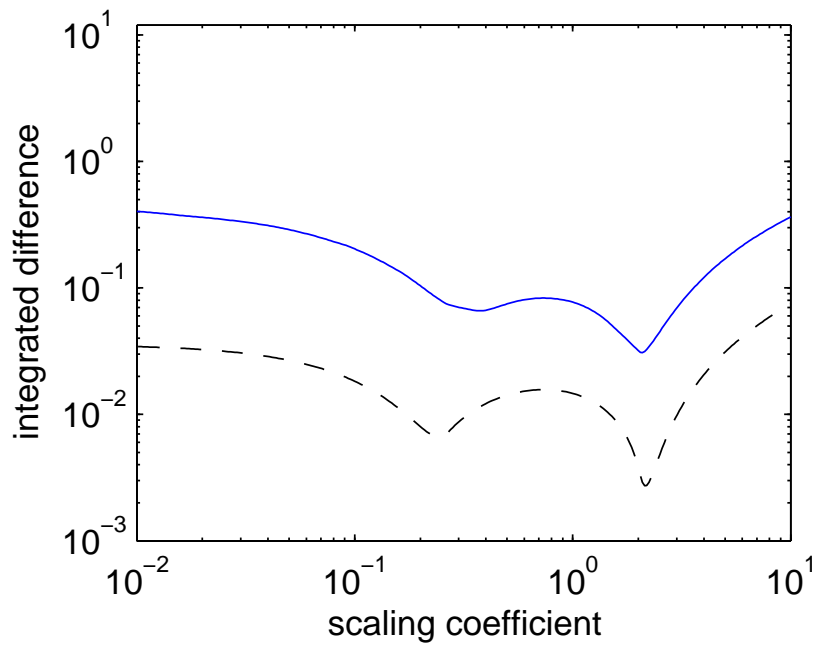

FIGURE 10. Integrated difference in horizontal (continuous line) and vertical (dashed line) velocities between mixed Stokes-Darcy and Darcy-Brinkman results as a function of the scaling coefficient $c$ for a corner flow in a fluid overlying a porous layer with a linear porosity $\left(D a=10^{-4}\right.$ and $\left.\chi_{0}=0.5\right)$.

\subsection{Simplified physical model}

We consider a binary alloy in the corner-flow geometry with the boundary conditions introduced in the previous section, and we further suppose that the incoming fluid at $z=h$ has a fixed temperature $T_{\infty}$ and a fixed concentration $C_{\infty}$, while the temperature of the lower boundary at $z=0$ is fixed just above the eutectic temperature $T_{e}$. A mushy layer then grows from this lower boundary, leading to a time-evolving porosity field. Following classical studies in solidification, we suppose that the whole system is pulled vertically at a constant rate $V_{\text {pull }}$ and we look for a stationary solution in the moving frame of reference.

The solidifying binary alloy is fully characterised by 5 independent variables: the average enthalpy $h$; the average species concentration $C$; the two-dimensional Darcy velocity 
vector $\mathbf{u}$; and the liquid fraction $\chi$. These 5 variables are determined by 5 coupled equations characterising the transport of enthalpy, species, mass and momentum, as well as the thermodynamic constraint imposed by the phase diagram. Three other useful variables can be derived from these primary unknowns, i.e. the temperature $T$, the liquid enthalpy $h_{l}$ and the liquid concentration $C_{l}$.

We denote by $k_{k}$ the thermal conductivity, $c_{p, k}$ the heat capacity per unit volume and $D_{k}$ the species diffusivity of the liquid $(k=l)$ and the solid $(k=s)$ respectively. To keep the problem simple, we make the following assumptions. We neglect variations of material properties inside the control volume $\Delta V$, and suppose that the solid and the liquid have the same physical properties, i.e. $\left[\rho_{l}\right]^{l}=\left[\rho_{s}\right]^{s}=\rho,\left[k_{l}\right]^{l}=\left[k_{s}\right]^{s}=k$, and $\left[c_{p, l}\right]^{l}=\left[c_{p, s}\right]^{s}=c_{p}$. We neglect species diffusion, i.e. $\left[D_{l}\right]^{l}=\left[D_{s}\right]^{s}=0$. We suppose that the temperature is constant inside the control volume $\Delta V$, i.e. $\left[T_{l}\right]^{l}=\left[T_{s}\right]^{s}=T$. We suppose that the liquid is well mixed inside the control volume $\Delta V$, i.e. $\left[C_{l}\right]^{l}=$ $C_{l}$. Besides, we consider a simplified equilibrium phase diagram, where the solidus and liquidus curves are respectively given by

$$
\begin{gathered}
C=0 \quad \text { or } \quad T_{\text {solidus }}(C)=T_{e}, \\
T_{\text {liquidus }}(C)=T_{m}-\frac{T_{m}-T_{e}}{C_{e}} C,
\end{gathered}
$$

where $C_{e}$ corresponds to the eutectic concentration and $T_{m}$ to the liquidus temperature at $C=0$.

In the corner-flow geometry, we look for a solution in which the enthalpy, temperature, concentrations, liquid fraction, and velocity function $f$ depend on time $t$ and depth $z$ only. We define the dimensionless temperature and concentration by

$$
\theta=\frac{T-T_{e}}{T_{\infty}-T_{e}}, \quad C=\frac{C-C_{e}}{C_{\infty}-C_{e}}
$$

and introduce the characteristic scales for energy $c_{p}\left(T_{\infty}-T_{e}\right)$, length $h$, time $h^{2} / \kappa$, where $\kappa=k / c_{p}$, velocity $\kappa / h$ and pressure $\eta \kappa / h^{2}$. Then, the dimensionless mesoscopic transport equations in the moving frame of reference, derived from their microscopic counterpart using the volume-averaging method (see for instance Beckermann \& Viskanta 1988), are:

$$
\begin{gathered}
\frac{\partial h}{\partial t}+\left(f-V_{\text {pull }}\right) \frac{\partial h}{\partial z}-\frac{\partial^{2} h}{\partial z^{2}}=L f \frac{\partial \chi}{\partial z}-L \frac{\partial^{2} \chi}{\partial z^{2}} \\
\frac{\partial C}{\partial t}+\left(\frac{f}{\chi}-V_{\text {pull }}\right) \frac{\partial C}{\partial z}-\frac{1}{\chi^{2}} f \frac{\partial \chi}{\partial z} C=\frac{1}{\chi^{2}} f \frac{\partial \chi}{\partial z} \frac{C_{e}}{C_{\infty}-C_{e}}
\end{gathered}
$$

where

$$
h_{l}=L+\theta, \quad h=\chi L+\theta,
$$

and since we suppose here that we remain strictly above the eutectic temperature,

$$
C=\chi C_{l}+(\chi-1) \frac{C_{e}}{C_{\infty}-C_{e}} .
$$

To close the problem, we finally translate the relationships given by the phase diagram in terms of enthalpy. For any given $(C, h)$, we compute

$$
h_{\text {liquidus }}(C)=L+m C,
$$

where $m$ is the dimensionless liquidus slope

$$
m=\frac{T_{m}-T_{e}}{T_{\infty}-T_{e}} \frac{C_{\infty}-C_{e}}{-C_{e}} .
$$


Then, if $h \geq h_{\text {liquidus }}(C), \chi=1$; else, since we remain strictly above the eutectic, the temperature and liquid concentration are related by the liquidus

$$
\theta=m C_{l},
$$

and from (5.5) and (5.6), $\chi$ is the solution between 0 and 1 of the second-order equation

$$
h=\chi L+m\left[\frac{C}{\chi}+\left(\frac{1}{\chi}-1\right) \frac{C_{e}}{C_{\infty}-C_{e}}\right] .
$$

One can notice that using the enthalpy and bulk concentration instead of the temperature and liquid concentration in the transport equations prevents us from calculating $\partial \chi / \partial t$ directly: rather, $\chi$ is determined using the known values of $h$ and $C$ and the phase diagram.

\subsection{Solution strategy and numerical approach}

Our initial state at $t=0$ is defined by $\theta=1$ except $\theta(0)=0, C=1$, and $\chi=1$ except $\chi(0)=1+\left(C_{\infty}-C_{e}\right) / C_{e}$, which comes from (5.6) with $C_{l}(0)=\theta(0) / m=0$. Hence, $h=L+1$ except $h(0)=L\left(1+\left(C_{\infty}-C_{e}\right) / C_{e}\right)$. We then progressively increment time until reaching the steady state. Knowing all fields at time $t^{n}$, we successively compute all fields at time $t^{n+1}=t^{n}+\Delta t$ in four successive steps. We first compute $h^{n+1}$ using a time-implicit space-centered discretisation of (5.3) with the boundary conditions

$$
h_{z=1}^{n+1}=L+1 \text { and } h_{z=0}^{n+1}=\chi_{z=0}^{n} L .
$$

We then compute $C^{n+1}$ using a time-implicit space-upwind discretisation of (5.4) with the boundary condition

$$
C_{z=1}^{n+1}=1
$$

From $\left(C^{n+1}, h^{n+1}\right)$, we compute $\chi^{n+1}$ using the process described in (5.7)-(5.10).

Neglecting advective terms as well as convective terms (i.e. $\rho_{l}$ does not depend on $\theta$ nor on $C_{l}$ ), we finally compute $f^{n+1}$ using $\chi^{n+1}$ and either the Darcy-Brinkman equation (4.4) or the mixed Stokes-Darcy equations (4.7a) and (4.16). As in the previous section, since $\chi \rightarrow 1$ at the interface, only our interfacial conditions can be used with the CarmanKozeny permeability. At each time step, we then compute $z_{\delta}^{n+1}$, the interfacial position for velocity boundary conditions, using local values of porosity and permeability, i.e.

$$
z_{\delta}^{n+1}=z_{\chi}^{n+1}-c \sqrt{\frac{\Pi^{n+1}\left(z_{\delta}^{n+1}\right) / h^{2}}{\chi^{n+1}\left(z_{\delta}^{n+1}\right)}},
$$

where $c$ is a scaling constant and $z_{\chi}^{n+1}$ the position of the liquid-porous matrix interface. However, in order to compare with previous multiple-domain studies of solidification (e.g. Schulze \& Worster 1999), we also present here results computed with either a continuous tangential velocity at $z_{\chi}^{n+1}$ or Beavers \& Joseph (1967) interfacial conditions at $z_{\chi}^{n+1}$, using a non-divergent permeability function

$$
\hat{\Pi}=\hat{\Pi}_{0} \chi^{3} .
$$

In these cases, $\hat{\Pi}_{0}$ is chosen such that $\Pi(z=0)=\hat{\Pi}(z=0)$.

\subsection{Results}

Typical steady-state profiles for Darcy-Brinkman and mixed Stokes-Darcy formulation with our interfacial conditions are presented in figure 11. One can notice that the steadystate porosity profile is close to linear. Hence, as previously, velocities given by the limit 
$c \rightarrow 0$ are unrealistic, whereas $c \sim 1$ gives a qualitatively good agreement between singledomain and multiple-domain formulations. The simple case of solidification studied here demonstrates that the predicted macroscopic bulk concentration field is very sensitive to the choice of interfacial conditions: significant variations take place throughout the whole depth of the formed solid. This is mainly due to the assumption of no species diffusion: the bulk concentration is only advected, and thus highly sensitive to the velocity field. In most natural and industrial settings, the Lewis number (i.e. the ratio of thermal to species diffusivity) is large and this conclusion will remain valid. As shown in figure 12, the agreement between the two formulations can be adjusted through the choice of the scaling coefficient $c$. It is not possible to choose a value that gives simultaneously the best agreement for all macroscopic fields, but values of $c \sim 2-4$ work reasonably well.

Steady-state profiles obtained with the mixed Stokes-Darcy formulation with a continuous tangential velocity or Beavers \& Joseph (1967) conditions at the liquid-porous interface are presented in figure 13. Significant differences in all macroscopic fields between these formulations and Darcy-Brinkman formulation are then observed through the whole depth of the system, which can be adjusted neither by the scaling coefficient $\alpha$ nor by the permeability function.

Special care should thus be taken in the choice of the microscopic fluid-porous matrix interface conditions when trying to determine realistic macrosegregation patterns of binary alloy solidification in industrial or natural settings. Differences calculated here with a simplified physical model would be even larger when taking into account buoyancy effects, since then velocities and bulk concentration would actively interact. Direct comparison with experiments is necessary to validate a given approach.

\section{Conclusion}

Studies of multiphase flows can be divided into two groups. Some models use a single Darcy-Brinkman formulation for both the liquid and the porous domains, and resolve the system on a single mesoscopic scale. Other models use independent sets of equations for the liquid and the porous matrix, with imposed boundary conditions at the sharp interface.

Previously suggested interfacial conditions, written directly at the porous matrixliquid interface, do not give a satisfactory agreement with Darcy-Brinkman results, even in the simple configurations studied here. Indeed, Darcy's equation does not take into account fluid-fluid viscous interactions that become important very close to the interface and/or when the permeability becomes large. We therefore define a viscous transition zone below the liquid-porous interface, inside the porous matrix, where Stokes law still applies, and consider Darcy's law only below it. This transition zone is of the order of the square root of the local value of the permeability divided by the liquid fraction. Using this formulation, a good agreement with Darcy-Brinkman results is obtained, even for a continuous transition between liquid and solid, for instance during binary alloy solidification.

We must however keep in mind that the derivation of the mesoscopic volume-average Darcy-Brinkman equation necessitates lots of approximations. The study proposed here does not demonstrate its validity, which should be tested by careful experimental investigations (see also Zhao \& Chen 2001). We can for instance suggest to reproduce Beavers \& Joseph (1967) experiments in the corner geometries studied here or to investigate precisely the macrosegregation patterns produced by binary alloy solidification, since this would simultaneously validate the Darcy-Brinkman equation and test the various suggested interfacial boundary conditions in the Stokes-Darcy approach. The approach 

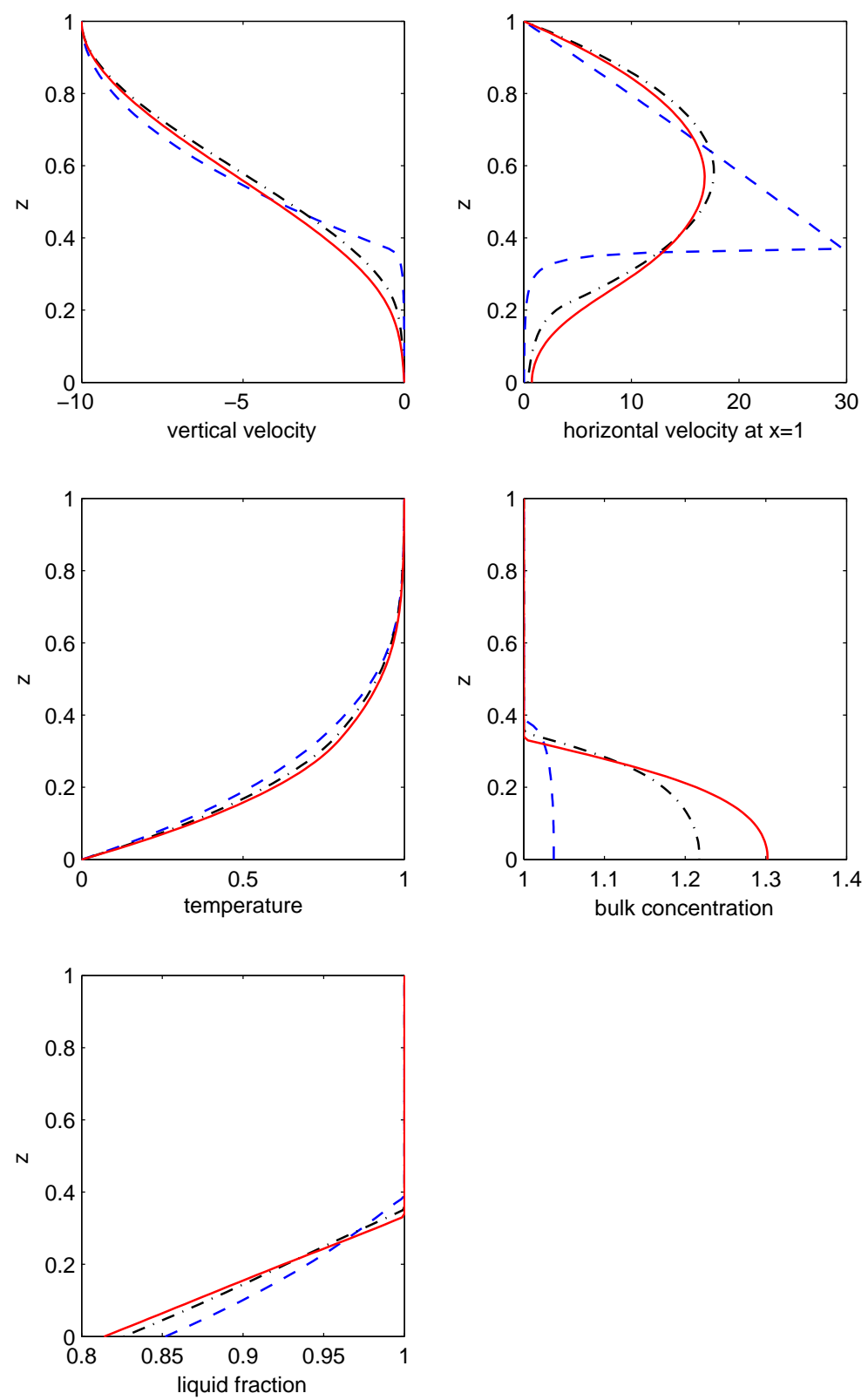

FiguRE 11. Comparison between steady-state profiles given by Darcy-Brinkman formulation (continuous line) and mixed Stokes-Darcy formulation with our interfacial conditions for the solidification of a binary alloy in a corner flow ( $V_{\text {pull }}=1, V=10, L=10, D a=10^{-4}$, $\left.C_{e} /\left(C_{\infty}-C_{e}\right)=-7, m=0.8\right)$. Dashed line: mixed Stokes-Darcy in the limit $c \rightarrow 0$; dashed-dotted line: mixed Stokes-Darcy with $c=1$.

formulated and illustrated in this paper will allow systematic comparisons to be made between single-domain and sharp-interface models of alloy solidification; comparisons which may, in turn, indicate the need for better modelling of the interfacial regions of mushy zones. 

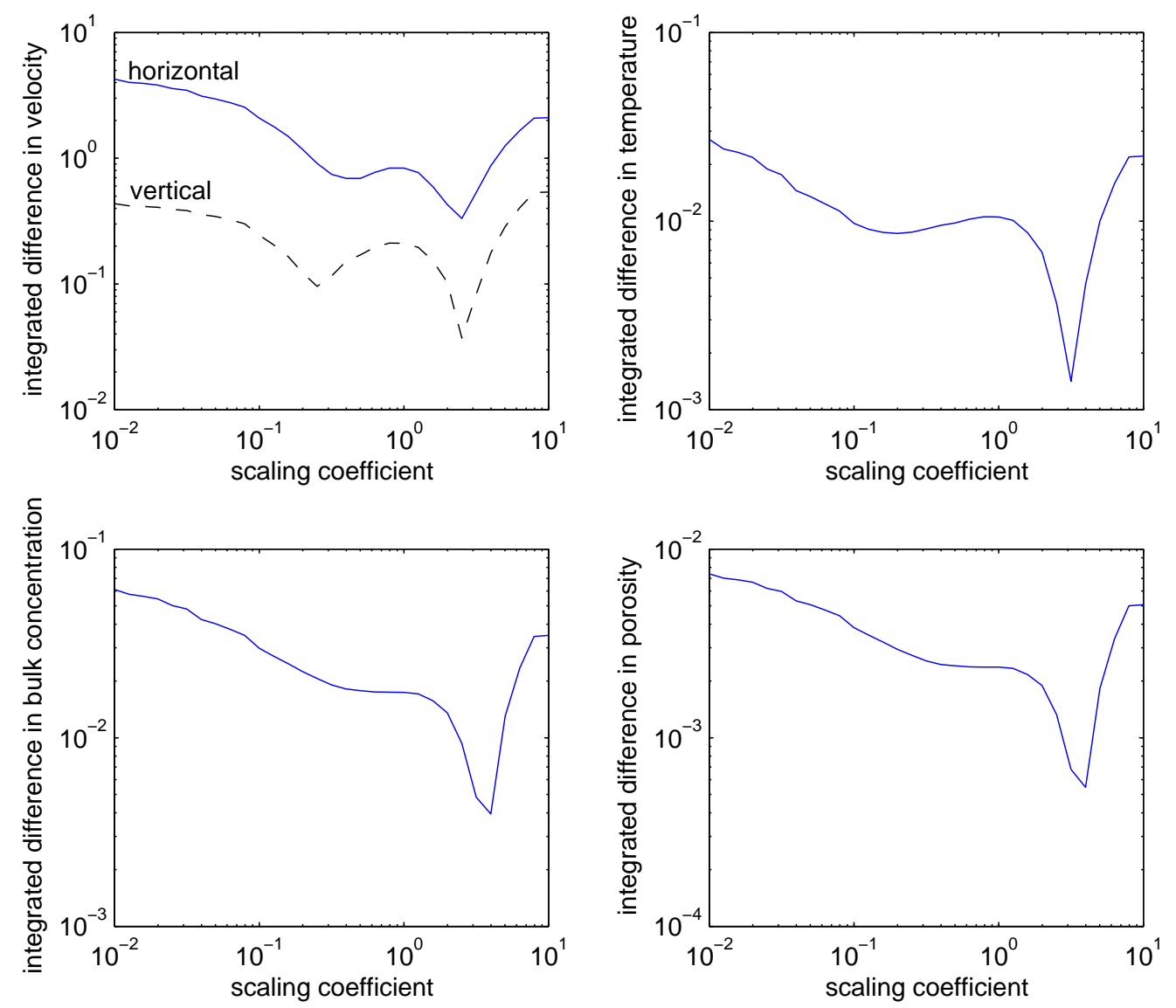

FIGURE 12. Integrated difference between Darcy-Brinkman and mixed Stokes-Darcy formulation with our interfacial conditions as a function of the scaling coefficient $c\left(V_{\text {pull }}=1, V=10\right.$, $\left.L=10, D a=10^{-4}, C_{e} /\left(C_{\infty}-C_{e}\right)=-7, m=0.8\right)$.

The authors would like to acknowledge support from the Leverhulme Trust and from the European Community (Marie Curie Intra European Fellowship FP6-501040).

\section{REFERENCES}

Batchelor, G. K. 2000 An Introduction to Fluid Dynamics. Cambridge University Press.

Bear, J. 1972 Dynamics of Fluids in Porous Media. Dover Publications.

Beavers, G. S. \& Joseph, D. D. 1967 Boundary conditions at a naturally permeable wall. $J$. Fluid Mech. 30, 197-207.

Beckermann, C., Diepers, H.-J., Steinbach, I., Karma, A. \& Tong, X. 1999 Modeling melt convection in phase-field simulations of solidification. J. Comp. Phys. 154, 468-496.

Beckermann, C. \& Viskanta, R. 1988 Natural convection solid/liquid phase change in porous media. Int. J. Heat Mass Transfer 31, 35-46.

Bennon, W. D. \& Incropera, F. P. 1987 A continuum model for momentum, heat and species transport in binary solid-liquid phase change systems. Int. J. Heat Mass Transfer 30, 2161-2171.

Brinkman, H. C. 1947 A calculation of the viscous force exerted by a flowing fluid on a dense swarm of particles. Appl. Sci. Res. A 1, 27-34.

Chung, C. A. \& Worster, M. G. 2002 Steady-state chimneys in a mushy layer. J. Fluid Mech. 455, 387-411. 

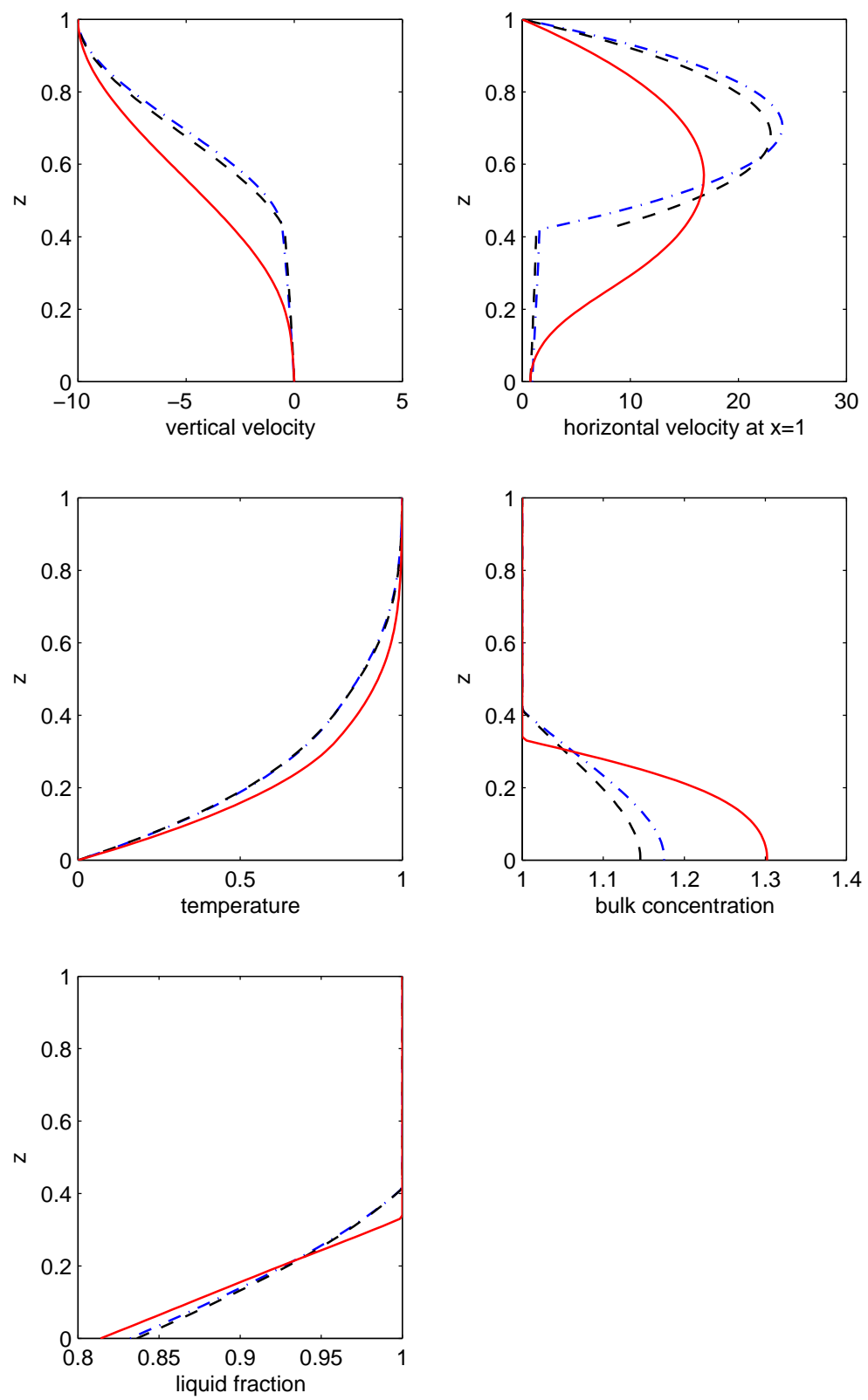

FIGURE 13. Comparison between steady-state profiles given by Darcy-Brinkman formulation (continuous line) and mixed Stokes-Darcy formulation using the non-divergent permeability function (5.14) and either a continuous horizontal velocity at the interface (dashed-dotted line) or Beavers $\&$ Joseph (1967) interfacial conditions with $\alpha=1$ (dashed line) for the solidification of a binary alloy in a corner flow $\left(V_{\text {pull }}=1, V=10, L=10, D a=10^{-4}\right.$ corresponding to $\left.\hat{\Pi}_{0} / h^{2}=2.9 \times 10^{-3}, C_{e} /\left(C_{\infty}-C_{e}\right)=-7, m=0.8\right)$.

Cieszko, M. \& Kubik, J. 1999 Derivation of matching conditions at the contact surface between fluid-saturated porous solid and bulk fluid. Transport in Porous Media 34, 319-336.

Felicelli, S. D., Heinrich, J. C. \& Poirier, D. R. 1991 Simulation of freckles during vertical solidification of binary alloys. Metall. Trans. B 22, 847-859. 
Gray, W. G. 1975 A deviation of the equations for multiphase transport. Chem. Eng. Sci. 30, $229-233$.

Gu, J. \& Beckermann, C. 1999 Simulation of convection and macrosegregation in a large steel ingot. Met. and Mat. Trans. A 30A, 1357-1366.

Hills, R. N., Loper, D. E. \& Roberts, P. H. 1983 A thermodynamically consistent model of a mushy zone. Q. J. Mech. Appl. Math. 36, 505-539.

KAEmpfer, T. U. \& RAPPAZ, M. 2003 Modelling of macrosegregation during solidification processes using an adaptive domain decomposition method. Modelling Simul. Mater. Sci. Eng. 11, 575-597.

Lesieur, M. 1993 Numerical simulations of turbulence in shear flows. In Applied Scientific Research, Advances in turbulence IV (ed. F. T. M. Nieuwstadt), pp. 345-351. Kluwer.

Levy, T. \& SAnchez-PAlencia, E. 1975 On the boundary conditions for fluid flow in porous media. Int. J. Engng. Sci. 13, 923-940.

Moin, P., Squires, K., Cabot, W. \& Lee, S. 1991 A dynamic subgrid-scale model for compressible turbulence and scalar transport. Phys. Fluids A3, 2746-2757.

Neale, G. H. \& NADER, W. K. 1974 Prediction of transport processes within porous media: Creeping flow relative to a fixed swarm of spherical particles. AIChE J. 20, 530-538.

OCHOA-TAPIA, J. \& WhitAKER, S. 1995 a Momentum transfer at the boundary between a porous medium and a homogeneous fluid: I-theoretical development. Int. J. Heat Mass Transf. 38, 2635-2646.

OChOA-TAPiA, J. \& Whitaker, S. $1995 b$ Momentum transfer at the boundary between a porous medium and a homogeneous fluid: Ii- comparison with experiment. Int. J. Heat Mass Transf. 38, 2647-2655.

Saffman, P. G. 1971 On the boundary condition at the surface of a porous medium. Studies in Appl. Math. L(2), 93-101.

Schneider, M. C., Gu, J. P., Beckermann, C., Boettinger, W. J. \& Kattner, U. R. 1997 Modeling of micro- and macrosegregation and freckle formation in single-crystal nickel-base superalloy directional solidification. Metall. Mater. Trans. A 28, 1517-1531.

Schulze, T. P. \& Worster, M. G. 1999 Weak convection, liquid inclusions and the formation of chimneys in mushy layers. J. Fluid Mech. 388, 197-215.

Shyy, W., Ouyang, H., Blosch, E., Thakur, S. S. \& Liu, J. 1997 Computational Techniques for Complex Transport Phenomena. Cambridge University Press.

WhitAkeR, S. 1999 The method of volume averaging. Kluwer Academic Publishers, Dordrecht, The Netherlands.

Worster, M. G. 1997 Convection in mushy layers. Ann. Rev. Fluid Mech. 29, 91-122.

Worster, M. G. 2000 Solidification of fluids. In Perspectives in Fluid Dynamics (ed. G. K. Batchelor, H. K. Moffat \& M. G. Worster), pp. 393-446. Cambridge University Press.

Zhao, P. \& Chen, C. F. 2001 Stability analysis of double-diffusive convection in superposed fluid and porous layers using a one-equation model. Int. J. Heat Mass Transfer 44, 46254633 .

\section{Appendix A. The volume averaging method}

We consider a domain comprising both a solid and a liquid phase, for instance a porous medium or a solidifying material, and define a mesoscopic volume $\Delta V$ large enough to smooth the morphological complexities, but small enough to capture the global transport properties (i.e. typically a few pore lengths). At this intermediate scale, mesoscopic transport equations can be derived from the volume averaging of the microscopic equations over $\Delta V$. Concepts and theorems that are used by this technique have been extensively studied (e.g. Gray 1975; Whitaker 1999) and are here briefly recalled for application to the momentum equation.

We define the liquid phase function $\nu_{l}$ by

$$
\nu_{l}= \begin{cases}1 & \text { in the liquid, } \\ 0 & \text { in the solid }\end{cases}
$$


and the liquid volume fraction (porosity) by

$$
\chi=\frac{1}{\Delta V} \int_{\Delta V} \nu_{l} d V=\frac{\Delta V_{l}}{\Delta V},
$$

where $d V$ is a infinitesimal element of volume relative to the control volume $\Delta V$ and $\Delta V_{l}$ the volume of liquid contained in $\Delta V$. The volume average of a given liquid quantity per unit volume $\phi_{l}$ is

$$
\left[\phi_{l}\right]=\frac{1}{\Delta V} \int_{\Delta V} \phi_{l} \nu_{l} d V .
$$

We also define the intrinsic volume average, i.e. the volume average in the liquid phase only

$$
\left[\phi_{l}\right]^{l}=\frac{1}{\Delta V_{l}} \int_{\Delta V} \phi_{l} \nu_{l} d V=\frac{\left[\phi_{l}\right]}{\chi},
$$

and the fluctuating component

$$
\hat{\phi}_{l}=\left(\phi_{l}-\left[\phi_{l}\right]^{l}\right) \nu_{l} .
$$

We now introduce three useful mathematical formulas, valid when functions change on typical scales larger than the averaging length (Gray 1975; Whitaker 1999):

- formula 1

$$
\left[\phi_{l} \psi_{l}\right]=\chi\left[\phi_{l}\right]^{l}\left[\psi_{l}\right]^{l}+\left[\hat{\phi}_{l} \hat{\psi}_{l}\right]
$$

where $\psi_{l}$ is another given liquid quantity per unit volume.

- formula 2

$$
\left[\frac{\partial \phi_{l}}{\partial t}\right]=\frac{\partial}{\partial t}\left[\phi_{l}\right]-\frac{1}{\Delta V} \int_{\Delta A} \phi_{l} \mathbf{w} \cdot \mathbf{n}_{\mathbf{l}} d A,
$$

where $\Delta A$ is the interfacial area between the liquid and solid phases in $\Delta V, \mathbf{w}$ is the velocity of the microscopic interface and $\mathbf{n}_{\mathbf{l}}$ is the outward unit normal vector of the infinitesimal area $d A$.

- formula 3

$$
\left[\nabla \phi_{l}\right]=\nabla\left[\phi_{l}\right]+\frac{1}{\Delta V} \int_{\Delta A} \phi_{l} \mathbf{n}_{\mathbf{l}} d A
$$

that can also be written

$$
\left[\nabla \phi_{l}\right]=\chi \nabla\left[\phi_{l}\right]^{l}+\frac{1}{\Delta V} \int_{\Delta A} \hat{\phi}_{l} \mathbf{n}_{l} d A .
$$

The demonstration of these three formulas is straightforward in the context of slowly variable functions, noting that

$$
\begin{aligned}
& \frac{\partial \nu_{l}}{\partial t}= \begin{cases}\mathbf{w} \cdot \mathbf{n}_{\mathbf{l}} & \text { on the liquid-solid interface } \\
0 & \text { elsewhere }\end{cases} \\
& \nabla \nu_{l}= \begin{cases}-\mathbf{n}_{\mathbf{l}} & \text { on the liquid-solid interface } \\
\mathbf{0} & \text { elsewhere }\end{cases}
\end{aligned}
$$

When functions change rapidly (i.e. over lengthscale comparable to the averaging length, as for instance in a sharp porous-liquid interface), a more complex approach is necessary (see for instance Ochoa-Tapia \& Whitaker 1995a). 